\title{
Comparison of molecular mechanisms of rheumatoid arthritis and osteoarthritis using gene microarrays
}

\author{
HONGQIANG LI", ZHENYONG HAO*, LIQIANG ZHAO, WEI LIU, \\ YANLONG HAN, YUNXING BAI and JIAN WANG
}

Department of Orthopedics, The Harbin Fifth Hospital, Harbin, Heilongjiang 150001, P.R. China

Received March 2, 2015; Accepted December 11, 2015

DOI: $10.3892 / \mathrm{mmr} .2016 .5144$

\begin{abstract}
The present study aimed to compare the molecular mechanisms of rheumatoid arthritis (RA) and osteoarthritis (OA). The microarray dataset no. GSE29746 was downloaded from Gene Expression Omnibus. After data pre-processing, differential expression analysis between the RA group and the control, as well as between the OA group and the control was performed using the LIMMA package in $\mathrm{R}$ and differentially expressed transcripts (DETs) with $\mid \log _{2}$ fold change (FC) $\mid>1$ and $\mathrm{P}<0.01$ were identified. DETs screened from each disease group were then subjected to functional annotation using DAVID Next, DETs from each group were used to construct individual interaction networks using the BIND database, followed by sub-network mining using clusterONE. Significant functions of nodes in each sub-network were also investigated. In total, 19 and 281 DETs were screened from the RA and OA groups, respectively, with only six common DETs. DETs from the RA and OA groups were enriched in 8 and 130 gene ontology (GO) terms, respectively, with four common GO terms, of which to were associated with phospholipase C (PLC) activity. In addition, DETs screened from the OA group were enriched in immune response-associated GO terms, and those screened from the RA group were largely associated with biological processes linked with the cell cycle and chromosomes. Genes involved in PLC activity and its regulation were indicated to be altered in RA as well as in OA. Alterations in the expression of cell cycle-associated genes were indicated to be linked with the occurrence of OA, while genes participating in the immune response were involved in the occurrence of RA.
\end{abstract}

Correspondence to: Professor Jian Wang, Department of Orthopedics, The Harbin Fifth Hospital, 27 Jiankang Road, Dongli, Harbin, Heilongjiang 150001, P.R. China

E-mail: jianwangjwjw@163.com

*Contributed equally

Key words: rheumatoid arthritis, osteoarthritis, differentially expressed transcripts, functional annotation, sub-network analysis

\section{Introduction}

Arthritis comprises a variety of diseases with joint pain as a common feature and includes osteoarthritis (OA), rheumatoid arthritis (RA) and psoriatic arthritis, while their underlying mechanisms are divergent (1). OA is a degenerative disease commonly manifesting with mechanical abnormalities of weight-bearing joints and hands, including knees and hips. It is characterized by loss of matrix proteoglycans, fibrillation of cartilage surface and eventual loss of collagenous matrix. Substantial studies have proven that synovial membrane inflammation, abnormal articular chondrocyte differentiation and bone remodeling contribute to the progression of OA (2-4). RA is a systemic autoimmune disease, in which the immune system targets body cells of the same organism. It features chronic inflammation of the synovium and subsequent cartilage destruction as well as bone erosion. The complement system is known to be involved in the induction and progression of inflammatory reactions in RA $(5,6)$.

Synovial fibroblasts (SFs), the most abundant resident cell type in human synovial tissue, are thought to have an important role in the pathogenesis of chronic arthritis (7) and display marked hyperplasia in OA and RA (8). Furthermore, alterations in the expression of various genes have also been observed to be associated with the phenotypic changes in OASFs and RASFs $(9,10)$. Previous microarray studies have confirmed a relatively high heterogeneity of the RASF phenotype $(11,12)$. By comparison between healthy SFs, RASFs and OASFs, Del Rey et al (13) found that OASFs possessed a more homogeneous phenotype compared to RASFs. The present study subjected the microarray data from Del Rey et al (13) to a bioinformatics analysis to identify common and differential molecular mechanisms underlying the two arthritis sub-types. The transcriptional expression profiles of the OASF and RASF samples were compared with those of SFs from healthy controls and key genes were identified.

\section{Materials and methods}

Microarray data. Gene microarray dataset GSE29746 was downloaded from Gene Expression Omnibus (http://www.ncbi. nlm.nih.gov/geo/) database (13). The data had been collected from SF cultures obtained from nine patients with RA, 11 age- and gender-matched patients with OA, and 11 age- and 

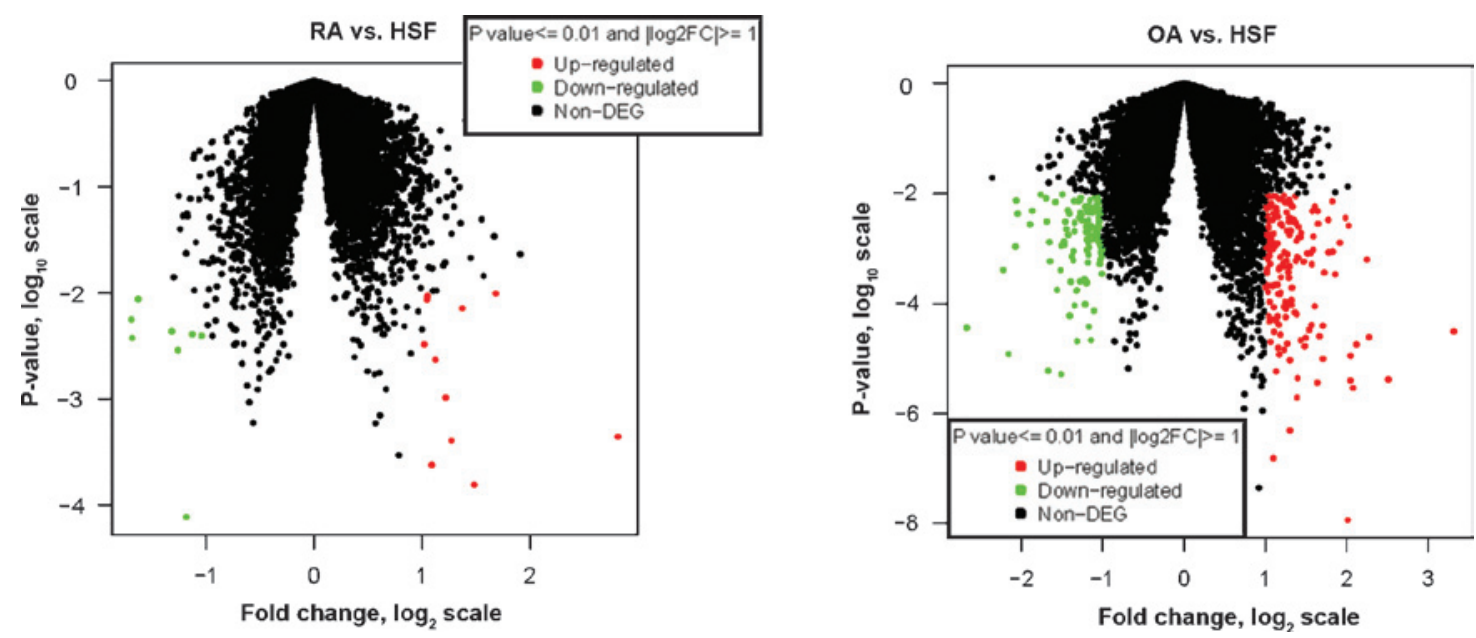

Figure 1. Volcano plots of expression values of all the transcripts. RA, synovial fibroblasts from rheumatoid arthritis group; OA, synovial fibroblasts from the osteoarthritis group; HSF, healthy synovial fibroblasts; DEG, differentially expressed gene.

gender-matched adult healthy donors. The platform was the Agilent-014850 Whole Human Genome Microarray 4x44 K G4112F (Agilent Technologies, Santa Clara, CA, USA).

Microarray data pre-processing. The gene expression profile data were extracted using the Linear Models for Microarray Data (LIMMA) package in R, followed by normexp background correction (14) and subsequent quantile normalization (15). The 95th quantile of all negative controls in each chip was calculated, and only probes with expression values larger than this value in all the samples were retained. According to the annotation platform, the values of probes corresponding to the same transcript were averaged and then defined as the final expression value of a transcript.

Screening of differentially expressed transcripts (DETS). Volcano plots were drawn to display the differential expression profiles of transcripts for each disease group and the control group. Differential expression analysis of transcripts between the RA group and the control group was performed using the $t$-test with the LIMMA package, as well as between the OA group and the control group (16). Transcripts with $\mid \log _{2}$ fold change (FC) $\mid>1$ and $\mathrm{P} \leq 0.01$ were screened as DETs. DETs from the two disease groups were compared, and a Venn diagram was used to display the result. A heatmap was also used to exhibit the overall expression profiles of DETs across all samples.

Functional annotation of DETs. The screened DETs were submitted to the online tool Database for Annotation, Visualization and Integrated Discovery (DAVID; http://david. abcc.ncifcrf.gov/summary.jsp) to perform functional annotation based on the gene ontology (GO) database using Fisher's exact test (17).

Analysis of interaction networks of DETs. DETs screened from each disease group were submitted to the Search Tool for the Retrieval of Interacting Genes/Proteins (http://www.string-db. org/) to construct protein-protein interaction (PPI) networks based on the Biomolecular Interaction Network Database (18).

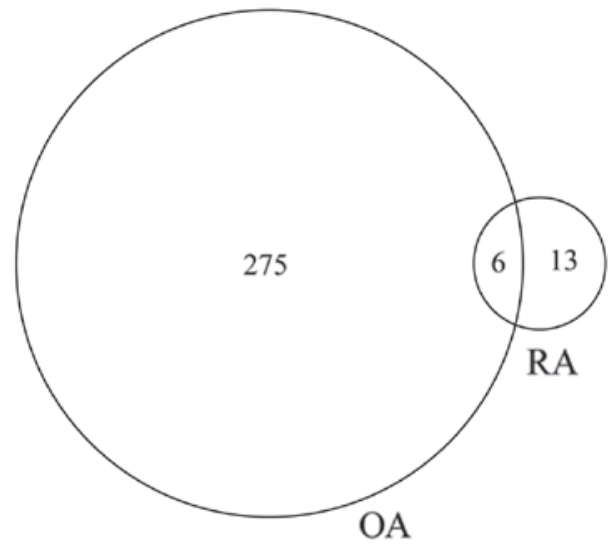

Figure 2. Venn diagram of differentially expressed transcripts between the rheumatoid arthritis group and the osteoarthritis group. RA, synovial fibroblasts from rheumatoid arthritis group; OA, synovial fibroblasts from the osteoarthritis group.

The sub-networks within each network were then detected by clustering analysis using clusterONE (19), and genes involved in each cluster were then subjected to functional enrichment analysis with DAVID ( $\mathrm{P}<0.05$ as cut-off value).

\section{Results}

Screening of differentially expressed transcripts. The differential expression profiles between each disease group and the control group were displayed in Volcano plots (Fig. 1). In total, 19 DETs were screened from the RA group, with $\log _{2} \mathrm{FCl}$ values of 1-2.8, among which eight genes were downregulated and 11 genes were upregulated. Furthermore, 281 DETs were screened from the OA group, with $\log _{2} \mathrm{FCl}$ values of 1.0-3.3, among which 113 genes were downregulated and 168 were upregulated. As illustrated by the Venn diagram (Fig. 2), the OA group contained a greater number of DETs than the RA group, while the two groups had six DETs in common (Table I). In addition, the heatmaps indicated that the disease samples may be separated from the control samples using the identified DETs (Fig. 3). 
Table I. Common differentially expressed transcripts between the RA group and the OA group.

\begin{tabular}{|c|c|c|c|c|c|}
\hline \multirow[b]{2}{*}{ Transcript } & \multirow[b]{2}{*}{ Gene symbol } & \multicolumn{2}{|c|}{ RA group } & \multicolumn{2}{|c|}{ OA group } \\
\hline & & $\log \mathrm{FC}$ & P-value & $\log \mathrm{FC}$ & P-value \\
\hline NM_182734 & PLCB1 & -1.18252 & $7.74 \times 10^{-5}$ & -1.03053 & $7.02 \times 10^{-3}$ \\
\hline NM_000211 & $I T G B 2$ & 2.81476 & $4.41 \times 10^{-4}$ & 3.31223 & $3.08 \times 10^{-5}$ \\
\hline NM_001853 & COL9A3 & -1.12924 & $4.07 \times 10^{-3}$ & -1.03848 & $5.18 \times 10^{-3}$ \\
\hline NM_139125 & $M A S P 1$ & -1.31698 & $4.33 \times 10^{-3}$ & -1.27800 & $3.61 \times 10^{-3}$ \\
\hline NM_002522 & NPTX1 & -1.69186 & $5.62 \times 10^{-3}$ & -1.48981 & $9.55 \times 10^{-3}$ \\
\hline NM_014638 & PLCH2 & 1.37193 & $7.12 \times 10^{-3}$ & 1.29122 & $7.54 \times 10^{-3}$ \\
\hline A_32_P4882 & $N A$ & -1.62833 & $8.70 \times 10^{-3}$ & -2.66329 & $3.58 \times 10^{-5}$ \\
\hline
\end{tabular}

RA, the rheumatoid arthritis; OA, the osteoarthritis; FC, fold change.

A

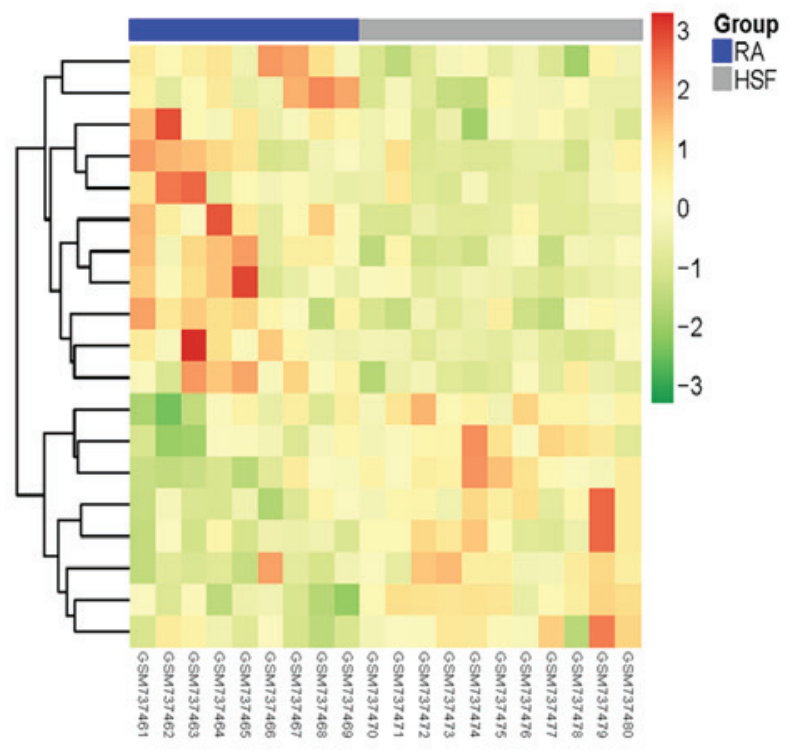

B

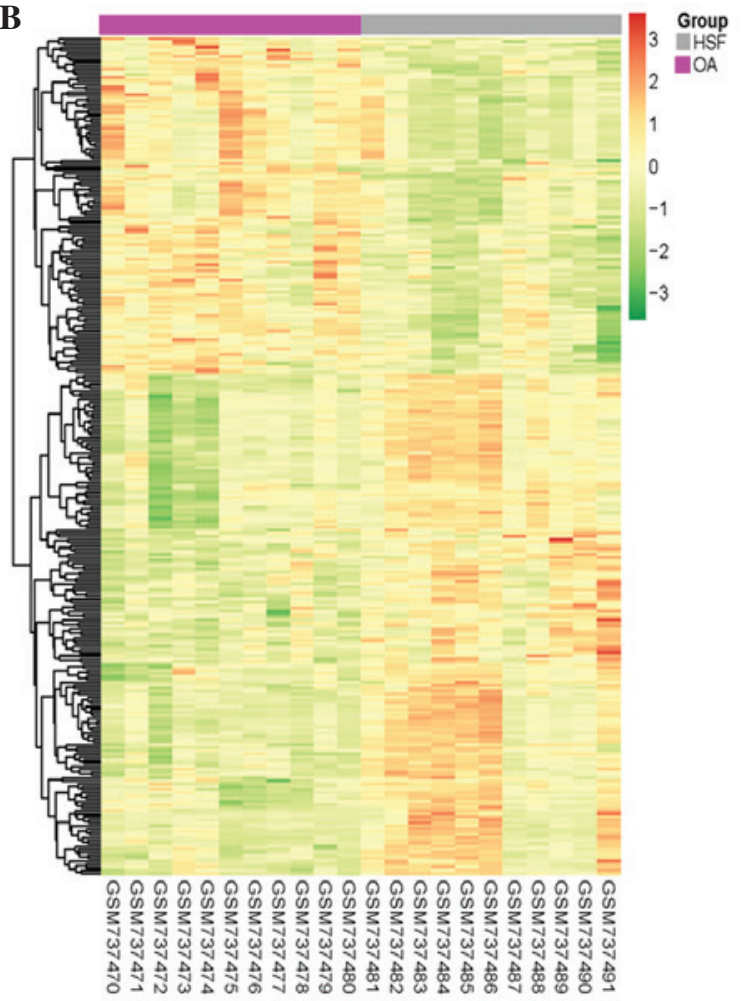

Figure 3. Clustering analysis of samples based on the screened differentially expressed transcripts. (A) Clustering analysis was based on the expression levels of 19 transcripts differentially expressed between the RA group and the healthy controls. (B) Clustering analysis based on the expression levels of 281 transcripts differentially expressed between the synovial fibroblasts in the OA group and the healthy controls. Each row represents a single gene and each column represents a tissue sample. The colored bar at the top indicates the group: Gray, HSF; blue, synovial fibroblasts from RA group; pink, synovial fibroblasts from OA group. Expression levels were indicated in a heat-map style with dark red indicating high expression and dark green low expression. RA, rheumatoid arthritis; OA, osteoarthritis; HSF, healthy synovial fibroblasts.

Functional annotation of DETs from each disease group. According to functional annotation, DETs from the RA and OA groups were enriched in 8 and 130 GO terms, respectively. Certain DETs from the RA group (e.g. PLCH2, PLCB1, NPTX1 and MASP1) and from the OA group (e.g. F2RL2, $P L C B 4, P L C H 2, P L C B 1$ and $P L C X D 1$ ) were commonly enriched in four GO terms, including two molecular function terms associated with phospholipase $\mathrm{C}$ activity (Table II). In addition to the four common GO terms, DETs screened from the RA patients were also associated with immune response (e.g. MASP1, IL27RA and ITGB2), while those from the OA group were predominantly associated with the cell cycle and chromosomes, including $N E K 2$, TTK, PTTG2, MAF, CENPN and SGOL2.

Analysis of PPI networks of DETs. The interaction networks of DETs from the RA group and the OA group are shown in Figs. 4 and 5, respectively. It was discovered that the PPI network based on DETs from the OA group was more complex than that of the RA group. The interaction network of the RA 
Table II. Common GO terms between the RA group and the OA group.

Differentially expressed genes

GO term and function

RA group

OA group

GO:0004435 - Phosphoinositide

phospholipase $\mathrm{C}$ activity

GO:0004629 - Phospholipase C activity

GO:0005509 - Calcium ion binding

GO:0007267 - Cell-cell signaling
PLCH2, PLCB1

PLCH2, PLCB1

NPTX1, MASP1,

PLCH2, PLCB1

NPTX1, WISP1, ITGB2
F2RL2, PLCB4, PLCH2, PLCB1

F2RL2, PLCB4, PLCH2, PLCB1, PLCXD1

F10, MASP1, NRXN2, PCDHB3, PCDHB2, VWCE, COLEC12, GRIN3A, C9ORF140, SLIT2, NPTX1, CDH15, CLGN, PLCB4, HPSE, PLCH2, PCDHB16, PLCB1, THBS1, DTNA

FGF18, ACHE, NTF3, PCDHB3, DLGAP5, EFNB2, MME, PCDHB2, ITGB2, RIMS1, NPTX1, PCDHB16, DTNA, HTR2A

RA, rheumatoid arthritis; OA, the osteoarthritis; GO, gene ontology.

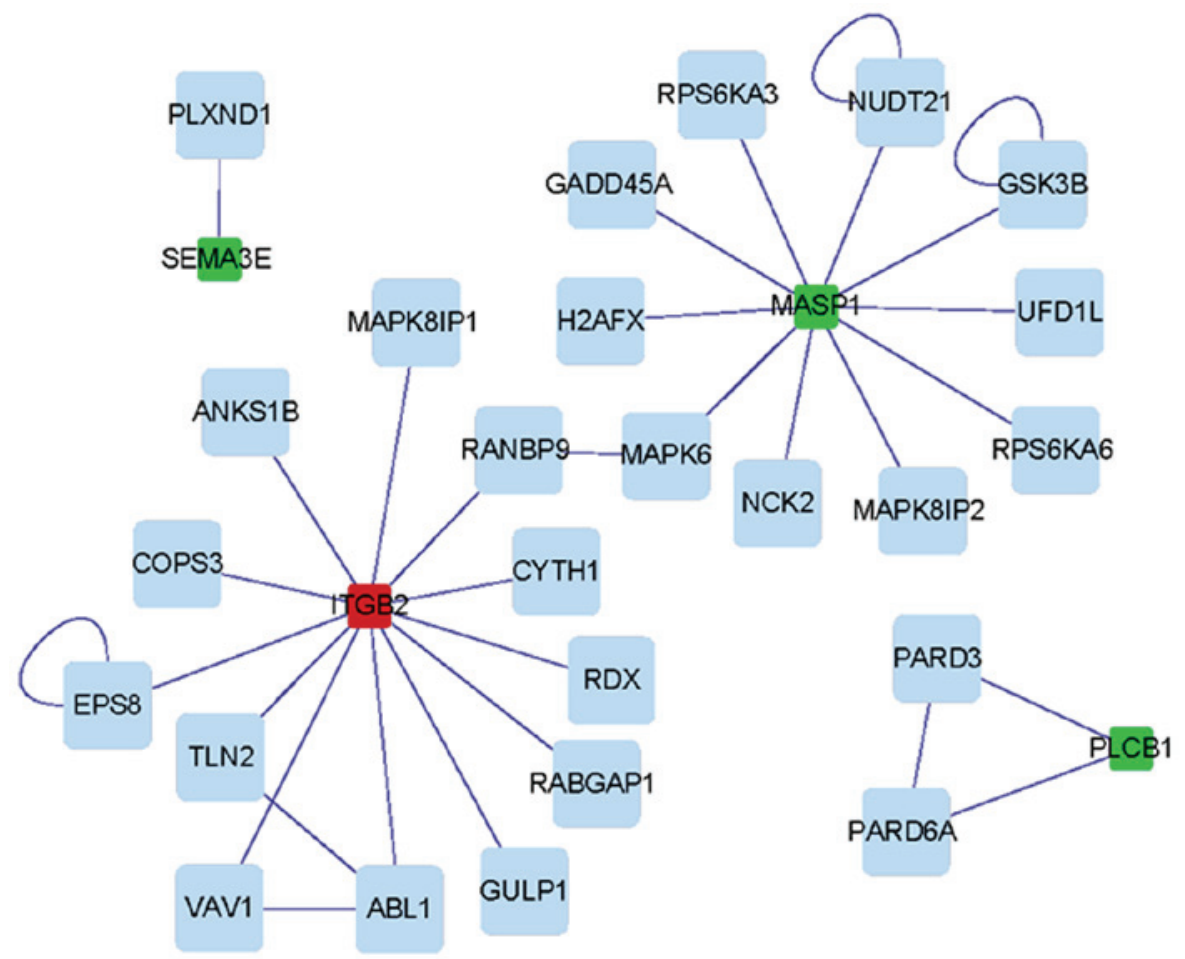

Figure 4. Interaction network of differentially expressed transcripts screened from synovial fibroblasts of patients with rheumatoid arthritis. Green squares represent downregulated DEGs and red squares indicate upregulated DEGs. DEG, differentially expressed gene.

group contained one sub-network, while that of the OA group had four sub-networks. DEGs including PSMB9, MAF, HTR $2 B$ and $H T R 2 A$ were the hubs of corresponding PPI sub-networks of DEGs from the OA group, while PLCB1 was a hub of the PPI sub-network of DEGs from the RA group (Table III).

\section{Discussion}

The present study revealed that in OA patients, the number of DEGs was higher compared to that in RA patients, which was consistent with the findings of Del Rey et al (13). This previous study identified 2,050 DEGs, several of which were also identified in the screening performed in the present study, such as ITGB2, PIP4K2C and NRXN2, although with different magnitudes of differential expression. Notably, the present study aimed to unravel the mechanisms underlying RA and OA from the perspective of PPI by building PPI networks, the constuction of which was not conducted in the previous study by Del Rey et al (13).

Two DEGs, $P L C H 2$ and $P L C B 1$, screened from the RA patients as well as the OA patients, were enriched in two PLC (phospholipase C) activity-associated GO terms, which may 
Table III. Identified sub-networks and functional annotation.

\begin{tabular}{|c|c|c|c|c|c|}
\hline \multirow[b]{2}{*}{ Variable } & \multicolumn{4}{|c|}{ OA group } & \multirow{2}{*}{$\frac{\text { RA group }}{\text { Cluster1 }}$} \\
\hline & Cluster1 & Cluster2 & Cluster3 & Cluster4 & \\
\hline Nodes (n) & 4 & 4 & 3 & 6 & 3 \\
\hline Density & 1 & 0.667 & 0.667 & 0.6 & 1 \\
\hline Quality & 1 & 1 & 1 & 0.529 & 1 \\
\hline $\mathrm{P}$-value & 0.008 & 0.011 & 0.026 & 0.046 & 0.016 \\
\hline Genes & $\begin{array}{l}A B C D 3, P E X 19 \\
A B C D 2, A B C D 1\end{array}$ & $\begin{array}{l}B A C H 1, M A F \\
M A F B, A T F 4\end{array}$ & $\begin{array}{c}H T R 2 A, M P D Z, \\
H T R 2 B\end{array}$ & $\begin{array}{c}\text { NCOA3, ESR1, } \\
\text { NCOA1, PSMB9, } \\
\text { GRIP1, ESR2 }\end{array}$ & $\begin{array}{l}\text { PLCB1, } \\
\text { PARD3, } \\
\text { PARD6A }\end{array}$ \\
\hline $\begin{array}{l}\text { Top significant } \\
\text { GO terms }\end{array}$ & $\begin{array}{l}\text { Peroxisomal } \\
\text { membrane }\end{array}$ & $\begin{array}{l}\text { Sequence-specific } \\
\text { DNA binding }\end{array}$ & Serotonin binding & $\begin{array}{l}\text { Steroid hormone receptor } \\
\text { signaling pathway }\end{array}$ & $\begin{array}{l}\text { Occluding } \\
\text { junction }\end{array}$ \\
\hline
\end{tabular}

RA, rheumatoid arthritis; OA, osteoarthritis.

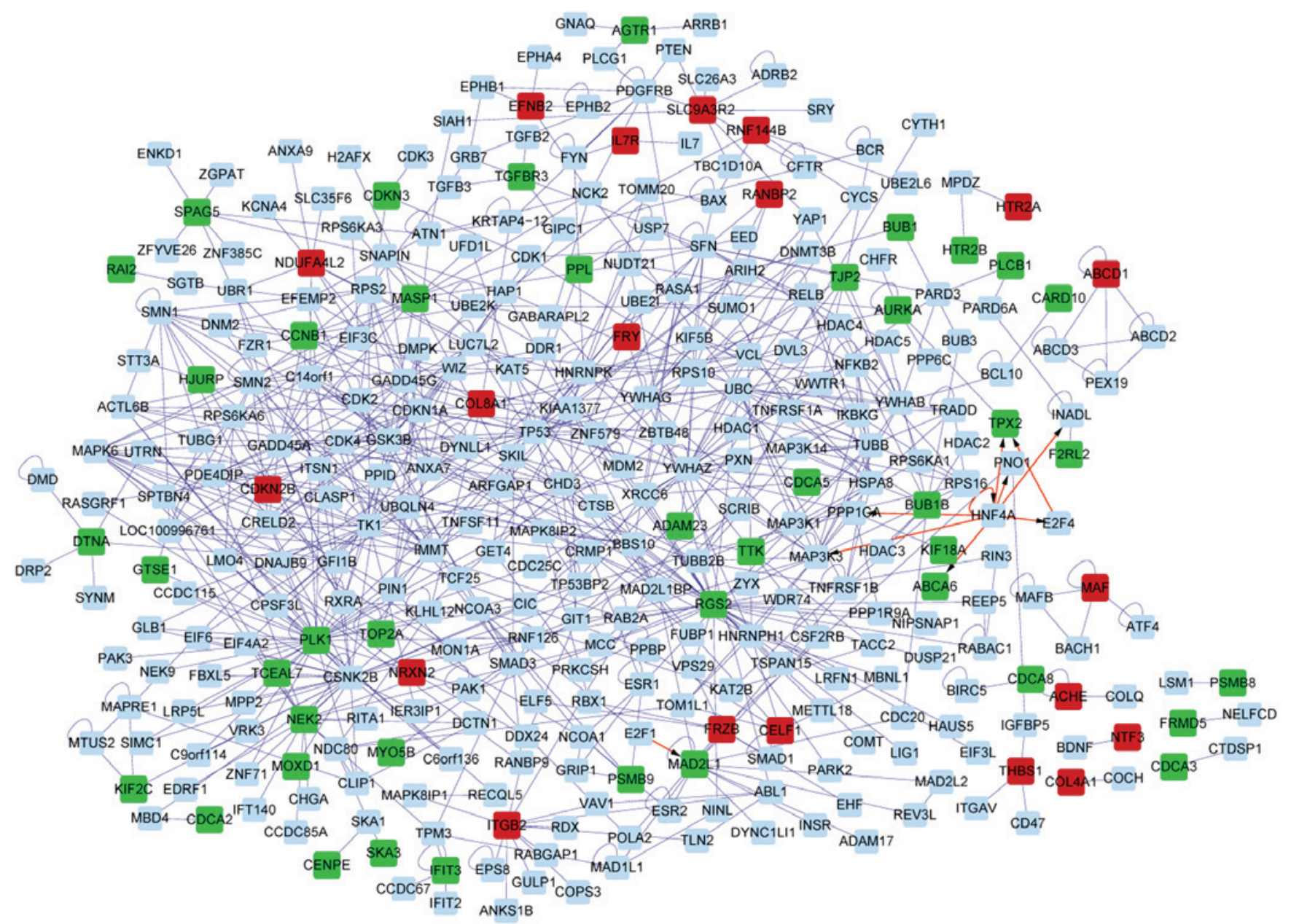

Figure 5. Interaction network of DEGs screened from synovial fibroblasts from patients in osteoarthritis group. Green squares represent downregulated DEGs and red squares indicate upregulated DEGs. DEG, differentially expressed gene.

infer that the two arthritis types share certain common mechanisms regarding phospholipase $\mathrm{C}$ activity. $\mathrm{PLCH} 2$ and $P L C B 1$ encode two members of the phosphoinositide-specific PLC superfamily, PLC-eta 2 and -beta 1, respectively, and PLC catalyzes the hydrolysis of phosphatidylinositol 4,5-bisphosphate into inositol 1,4,5-trisphosphate and 1,2-diacylglycerol) (20). Previous studies have reported a notable elevation of the pro-inflammatory enzyme PLA2 (phospholipase A2), another phospholipase type catalyzing the hydrolysis of membrane glycerophospholipids to release arachidonic acid and 
lysophospholipids in synovial fluids and sera of RA patients, and its expression has been proven to positively correlate with the disease activity in RA $(21,22)$. Vignon et al $(23)$ observed that PLA2 activity in RA and OA patients was similar, implying that pathological changes mediated by PLA2 are common in RA and OA. However, to the best of our knowledge, PLC activity has not been previously reported in RA or OA. As the two phospholipase types hydrolyze phospholipids at different sites, PLC may presumably also have a role in RA and OA, which requires further experimental validation.

Furthermore, in the present study, two PLC-regulating genes, HTR $2 B$ and HTR2A, which encode two members of the 5-hydroxytryptamine 2 receptor family that binds to the neurotransmitter serotonin, were also observed to be specifically differentially expressed in OA patients. These receptors activate PLC to initiate PLC-mediated signal transduction pathways (24). Of note, HTR2A was significantly upregulated, while $H T R 2 B$ was significantly downregulated, implying their different roles in regulating PLC. The two genes were observed to be enriched in the GO biological process terms phosphoinositide-mediated signaling and second-messenger-mediated signaling as well as in the cellular component term plasma membrane-associated processes. This suggests that alterations in PLC-associated biological functions may be the predominant aberrations in OA patients.

In addition, DEGs screened from OA samples were also specifically and predominantly enriched in GO terms associated with the cell cycle and chromosomes. Among the abundance of DEGs screened from the OA samples, PSMB 9 and $M A F$ were the key hubs of the sub-PPI network. PSMB9, which was downregulated in OA, encodes a member of the proteasome B-type family, and proteasomes cleave peptides via an adenosine triphosphate/ubiquitin-dependent pathway. This result was in accordance with previous studies, as the ubiquitin-proteasome pathway has been implicated in the pathogenesis of OA (25), and Rollin et al (26) has reported that PSMB9 is aberrantly expressed in chondrocytes of OA patients. As the present study indicated that PSMB 9 was mainly enriched in the GO biological process terms protein metabolic processes and cell cycle, it may be hypothesized that the downregulation of PSMB9 expression induces OA by disturbing normal protein metabolism and the cell cycle. $M A F$, a homolog of the avian musculoaponeurotic fibrosarcoma oncogene $V$-Maf, encodes a DNA-binding, leucine zipper-containing transcription factor. The increase in its expression in chondrocytes from patients with OA has been validated by Li et al (27), and it has been suggested that the upregulation of c-MAF expression may alter the phenotype of chondrocytes via regulating corresponding target genes (28), or interacting with other genes associated with chondrogenic differentiation (29). As in the present study, c-MAF was mainly enriched in chromosome-associated GO cellular component terms, it is presumed that it causes abnormal chondrogenic differentiation via inducing chromosomal abnormalities in OA patients.

Compared to those of OA, DETs screened from RA samples were exclusively enriched in immune response-associated GO biological process terms in addition to the four common GO terms, implying that the immune system has a particularly critical role in the occurrence of RA, which is consistent with the fact that RA is a systemic autoimmune disease. MASPl encodes a serine protease that functions as a component of the lectin pathway of complement activation (30). The upregulation of MASP1 expression, which was also observed by Rioja et al (31), indicated enhanced activation of the lectin pathway as well as the activation of the complement system in RA. Enhanced complement activation has been indicated to be potentially associated with the occurrence and/or augmentation of inflammation in RA (5). Activated synovial fibroblasts and upregulation of the expression of various adhesion molecules that mediate their attachment to the cartilage have been confirmed in rheumatoid arthritis (32). Among these adhesion molecules, the expression of integrin $\alpha$ group proteins, including VLA-3, -4 and -5 has been indicated to increase most significantly $(33,34)$. ITGB2 encodes integrin $\beta 2$, the expression of which was observed to be upregulated in the present study, which was also reported in RASFs by Del Rey et al (35); it is therefore indicated that ITGB2 is linked with RA.

In conclusion, the present study indicated that genes involved in PLC activity, including $P L C H 2$ and $P L C B 1$, and its regulation, including $H T R 2 A$ and $H T R 2 B$, are aberrantly expressed in RA as well as in OA. Alterations in the expression of genes associated with the cell cycle, including PSMB9 and $M A F$, were indicated to be linked with OA, while genes participating in the immune response were linked with RA.

\section{References}

1. Roivainen A, Söderström KO, Pirilä L, Aro H, Kortekangas P, Merilahti-Palo R, Yli-Jama T, Toivanen A and Toivanen P: Oncoprotein expression in human synovial tissue: An immunohistochemical study of different types of arthritis. Brit J Rheumatol 35: 933-942,1996.

2. Pullig O, Weseloh G, Ronneberger D, Käkönen SM and Swoboda B: Chondrocyte differentiation in human osteoarthritis: Expression of osteocalcin in normal and osteoarthritic cartilage and bone. Calcif Tissue Int 67: 230-240, 2000.

3. Drissi H, Zuscik M, Rosier R and O'Keefe R: Transcriptional regulation of chondrocyte maturation: Potential involvement of transcription factors in OA pathogenesis. Mol Aspects Med 26: 169-179, 2005.

4. Kwon DR and Park GY: Intra-articular injections for the treatment of osteoarthritis: Focus on the clinical use of several regimens. In: Osteoarthritis-Diagnosis, Treatment and Surgery. Chen Q (ed). InTech, Rijeka, pp67-100, 2012.

5. Nakagawa K, Sakiyama H, Tsuchida T, Yamaguchi K, Toyoguchi T, Masuda R and Moriya H: Complement C1 s activation in degenerating articular cartilage of rheumatoid arthritis patients: Immunohistochemical studies with an active form specific antibody. Ann Rheum Dis 58: 175-181, 1999.

6. Chen M, Daha MR and Kallenberg CG: The complement system in systemic autoimmune disease. J Autoimmun 34: J276-J286, 2010.

7. Bartok B and Firestein GS: Fibroblast-like synoviocytes: Key effector cells in rheumatoid arthritis. Immunol Rev 233: 233-255, 2010.

8. Izquierdo E, Cañete JD, Celis R, Del Rey MJ, Usategui A, Marsal S, Sanmartí R, Criado G and Pablos JL: Synovial fibroblast hyperplasia in rheumatoid arthritis: Clinicopathologic correlations and partial reversal by anti-tumor necrosis factor therapy. Arthritis Rheum 63: 2575-2583, 2011.

9. Stanczyk J, Ospelt C, Karouzakis E, Filer A, Raza K, Kolling C, Gay R, Buckley CD, Tak PP, Gay S and Kyburz D: Altered expression of microRNA-203 in rheumatoid arthritis synovial fibroblasts and its role in fibroblast activation. Arthritis Rheum 63: 373-381, 2011. 
10. Karouzakis E, Gay RE, Gay S and Neidhart M: Epigenetic control in rheumatoid arthritis synovial fibroblasts. Nat Rev Rheumatol 5: 266-272, 2009.

11. Van Baarsen LG, Wijbrandts CA, Timmer TC, Van Der Pouw Kraan TC, Tak PP and Verweij CL: Synovial tissue heterogeneity in rheumatoid arthritis in relation to disease activity and biomarkers in peripheral blood. Arthritis Rheum 62: 1602-1607, 2010.

12. Kasperkovitz PV, Timmer TC, Smeets TJ, Verbeet NL, Tak PP, van Baarsen LG, Baltus B, Huizinga TW, Pieterman E, Fero M, et al: Fibroblast-like synoviocytes derived from patients with rheumatoid arthritis show the imprint of synovial tissue heterogeneity: Evidence of a link between an increased myofibroblast-like phenotype and high-inflammation synovitis. Arthritis Rheum 52: 430-441, 2005.

13. Del Rey MJ, Usategui A, Izquierdo E, Cañete JD, Blanco FJ, Criado G and Pablos JL: Transcriptome analysis reveals specific changes in osteoarthritis synovial fibroblasts. Ann Rheum Dis 71: 275-280, 2012.

14. Ritchie ME, Silver J, Oshlack A, Holmes M, Diyagama D, Holloway A and Smyth GK: A comparison of background correction methods for two-colour microarrays Bioinformatics 23: 2700-2707, 2007.

15. Smyth GK and Speed T: Normalization of cDNA microarray data. Methods 31: 265-273, 2003.

16. Smyth GK: Limma: Linear models for microarray data. In: Bioinformatics and computational biology solutions using $\mathrm{R}$ and Bioconductor. Gentleman R, Carey VJ, Huber W, Irizarry RA, Dudoit S (eds). Springer, New York, pp397-420, 2005.

17. Huang da W, Sherman BT and Lempicki RA: Systematic and integrative analysis of large gene lists using DAVID bioinformatics resources. Nat Protoc 4: 44-57, 2009.

18. Franceschini A, Szklarczyk D, Frankild S, Kuhn M, Simonovic M, Roth A, Lin J, Minguez P, Bork P, von Mering C and Jensen LJ: STRING v9. 1: Protein-protein interaction networks, with increased coverage and integration. Nucleic Acids Res 41 (Database issue): D808-D815, 2013.

19. Nepusz T, Yu H and Paccanaro A: Detecting overlapping protein complexes in protein-protein interaction networks. Nat Methods 9: 471-472, 2012

20. McLaughlin S, Wang J, Gambhir A and Murray D: PIP(2) and proteins: Interactions, organization, and information flow. Annu Rev Biophys Biomol Struct 31: 151-175,2002.

21. Pruzanski W, Keystone EC, Sternby B, Bombardier C, Snow KM and Vadas P: Serum phospholipase A2 correlates with disease activity in rheumatoid arthritis. J Rheumatol 15: 1351-1355, 1988.

22. Lin M, Farewell V, Vadas P, Bookman AA, Keystone EC and Pruzanski W: Secretory phospholipase A2 as an index of disease activity in rheumatoid arthritis. Prospective double blind study of 212 patients. J Rheumatol 23: 1162-1166, 1996.
23. Vignon E, Balblanc JC, Mathieu P, Louisot P and Richard M: Metalloprotease activity, phospholipase A2 activity and cytokine concentration in osteoarthritis synovial fluids. Osteoarthritis Cartilage 1: 115-120, 1993.

24. Berg KA, Maayani S and Clarke WP: Interactions between effectors linked to serotonin receptors. Ann NY Acad Sci 861: 111-120,1998.

25. Jang BC, Sung SH, Park JG, Park JW, Bae JH, Shin DH, Park GY, Han SB and Suh SI: Glucosamine hydrochloride specifically inhibits COX-2 by preventing COX-2 N-glycosylation and by increasing $\mathrm{COX}-2$ protein turnover in a proteasome-dependent manner. $\mathrm{J}$ Biol Chem 282: 27622-27632, 2007.

26. Rollin R, Marco F, Camafeita E, Calvo E, López-Durán L, Jover J, López JA and Fernández-Gutiérrez B: Differential proteome of bone marrow mesenchymal stem cells from osteoarthritis patients. Osteoarthritis Cartilage 16: 929-935, 2008.

27. Li T, Xiao J, Wu Z and Qiu G: Over-expression of c-maf by chondrocytes in osteoarthritis. J Int Med Res 37: 129-135, 2009.

28. Maclean HE, Kim JI, Glimcher MJ, Wang J, Kronenberg HM and Glimcher LH: Absence of transcription factor c-maf causes abnormal terminal differentiation of hypertrophic chondrocytes during endochondral bone development. Dev Biol 262: 51-63, 2003.

29. Huang W, Lu N, Eberspaecher H and De Crombrugghe B: A new long form of c-Maf cooperates with Sox9 to activate the type II collagen gene. J Biol Chem 277: 50668-50675, 2002.

30. Goeldner I, Skare T, Boldt AB, Nass FR, Messias-Reason IJ and Utiyama SR: Association of MASP-2 levels and MASP2 gene polymorphisms with rheumatoid arthritis in patients and their relatives. PLoS One 9: e90979, 2014.

31. Rioja I, Clayton CL, Graham SJ, Life PF and Dickson MC: Gene expression profiles in the rat streptococcal cell wall-induced arthritis model identified using microarray analysis. Arthritis Res Ther 7: R101-R117, 2005.

32. Nikkari L, Aho H, Yli-Jama T, Larjava H, Jalkanen $M$ and Heino J: Expression of integrin family of cell adhesion receptors in rheumatoid synovium. Alpha 6 integrin subunit in normal and hyperplastic synovial lining cell layer. Am J Pathol 142: 1019-1027, 1993.

33. Rinaldi N, Schwarz-Eywill M, Weis D, Leppelmann-Jansen P, Lukoschek M, Keilholz U and Barth TF: Increased expression of integrins on fibroblast-like synoviocytes from rheumatoid arthritis in vitro correlates with enhanced binding to extracellular matrix proteins. Ann Rheum Dis 56: 45-51, 1997.

34. Ishikawa H, Hirata S, Andoh Y, Kubo H, Nakagawa N, Nishibayashi Y and Mizuno K: An immunohistochemical and immunoelectron microscopic study of adhesion molecules in synovial pannus formation in rheumatoid arthritis. Rheumatol Int 16: 53-60, 1996.

35. Del Rey MJ, Izquierdo E, Usategui A, Gonzalo E, Blanco FJ, Acquadro F and Pablos JL: The transcriptional response of normal and rheumatoid arthritis synovial fibroblasts to hypoxia. Arthritis Rheum 62: 3584-3594, 2010. 